\title{
SCHEDULING FOR INTERFERENCE MITIGATION USING ENHANCED INTERCELL INTERFERENCE COORDINATION
}

\author{
S. Gospel Ruben ${ }^{1}$,B. Jesvin Veancy ${ }^{2}$, P.Yogesh $^{3}$ \\ ${ }^{1}$ M.E Communication Systems, ${ }^{2}$ Assistant professor ( $\mathrm{Sl} \mathrm{Gr}$ ),Easwari Engineering College, India \\ ${ }^{3}$ Dept of Information Science and Technology, Anna University, Chennai, India
}

\begin{abstract}
Deployment of low power base station (pico) in macro cell is a key for providing high data rates. Cell range expansion is one of the features of LTE-advanced where low power base station in a heterogeneous network can increase their coverage area and neighboring cells can offload users to low power base station to reduce overload in macro cell. This presents a very challenging multi-user communication problem. Co-channel deployed Het-Net will experience interference between pico and macro. The main challenge of pico cells embedded into a macro cell is to let more users profit from the additional bandwidth introduced into the network. Enhanced Inter-cell Interference Coordination (eICIC) has been introduced to solve interference problem. Scheduling for eICIC is used to provide best solution for interference mitigation, overload in macro cell, fairness in resource usage by users. In this paper we have analyzed Round Robin scheduling for eICIC, results prove that fairness is maintained between users and throughput has been improved.
\end{abstract}

Key Words: LTE - Long term Evolution, UE - User Equipment, eNB - eNodeB.

\section{INTRODUCTION}

A heterogeneous network (HetNet) is a deployment of the conventional macro cell systems with small base station cells, pico. In heterogeneous networks, in addition to wellcoordinated high power macrocells, low-power cells such as pico, femto, and relays are underlaid in a macrocell area[12]. This approach consists of complementing the Macro layer with LPN (low power nodes) Pico base stations. This approach has been considered a way to improve the capacity and data rate in the areas covered by these low power nodes; they are mostly distributed in an unplanned manner depending on the areas that generate higher traffic[3]. Heterogeneous networks and specifically on the Pico base stations deployments that will be referred to as Pico-eNB. For small base stations with open access, those techniques offer the opportunity of relaxing the traffic demand at the macro-layer by offloading some of the macro user equipments (UEs) to the pico-layer. However, transmit power difference between macro and low power nodes is large enough to the users connected to the pico cell suffering from severe interference by a macro cell, which is one of major problems in LTE-heterogeneous network.

\section{PICO CELL RANGE EXPANSION}

In order to further increase the number of users offloaded from the macro cell, a technique of range extension is used to extend the Low Power Node coverage area and push more users to connect to the small cell [2]. A pico base station is regular eNB with lower transmit power compared to macro base stations and generally placed ad-hoc in the network. $* * *$

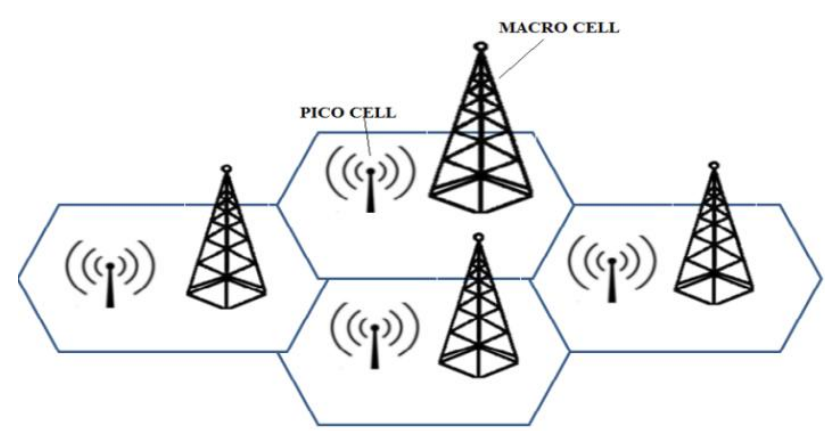

Fig-1: Heterogeneous network using Pico-eNBs

The deployment of these networks can cause large areas with low signal to interference values, resulting in a challenge to improve the performance of cell-edge users[4]. The huge difference between the transmit power levels of macro and small (e.g. pico) cells (around $15-20 \mathrm{dBm}$ ) implies a much smaller downlink coverage of a pico cell compared to that of the macro cell. Cell selection in LTE is based on terminal measurements of the received power of the downlink signal or more specifically the cell specific reference (CRS) downlink signaling. Due to the low signal strength least number of user moves to the pico cell and the increased number of users are present in the macro cell. 


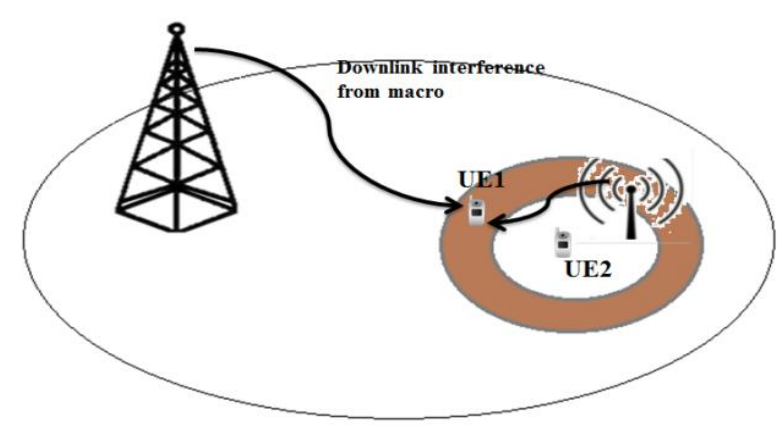

Fig-2:Range extension interference

\subsection{Interference associated with Range Extension}

In the increasing traffic demands of wireless data service the resources to cell border of pico cell provide unfairness in resource to mobile users which leads to the unfairness in load between the macro and the pico[6]. A high value of RE is desirable to get the most of the pico eNB. Addition and co-existence of cells with different size and scale introduce several interference that which is not managed appropriately, can degrade the the overall user throughput and coverage. Due to the difference in transmission powers of the MacroeNBs and the Pico-eNBs, in the range extension area, PicoeNB is selected by the terminal while the downlink power received by that terminal from the Macro-eNB is much higher than the power it receives from the Pico-eNB, this makes the users in the range extension area more prone to interference from the Macro-eNB.

\subsection{Enhanced Inter-Cell Interference Coordination}

Enhanced Inter-cell Interference Coordination (eICIC) is a framework by the 3GPP project to handle inter cell interference in Het-Net environments. In this approach transmissions from Macro-eNBs inflicting high interference onto Pico-eNBs users are periodically muted (stopped) during entire subframes, by this stategy, the Pico-eNB users that are suffering from a high level of interference from the aggressor Macro-eNB have a chance to be served[14]. Since RE pico UEs are only scheduled during mandatory ABS in the macro eNB, the number of mandatory ABS (i.e. TDM muting ratio) in the macro eNB should increase or decrease accordingly with the RE in the pico eNB and, consequently,with the number of cell-edge UEs in the cluster.

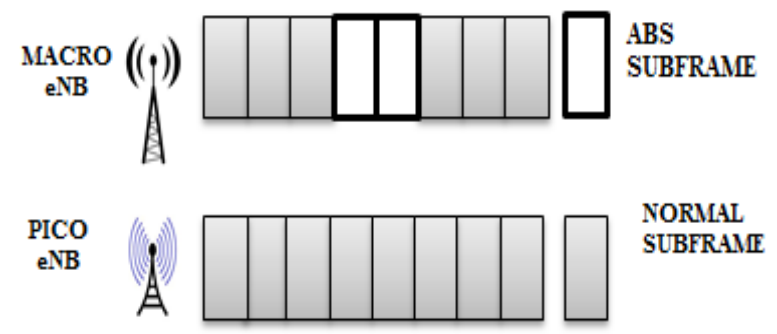

Fig-3: TDM eICIC for LTE-Advanced

\subsection{User cell selection and bias}

Cell selection by the user equipment is done by the user with respect to the maximum received signal strength[1]. Let the reference signal received power in $\mathrm{dBm}$ be $\mathrm{P}(\mathrm{i})$ from the cell as measured by the user equipment(UE) The user equipment selects the cell-S by

Where,

$$
\mathrm{S}=\arg \max (\mathrm{P}(\mathrm{i})+\alpha)
$$

$\alpha$-bias value.

Thus, by assigning larger bias to pico cell compared to macro cell, then one can ensure that the pico cell are not Underutilized[9].

\section{SCHEDULING ALGORITHMS}

Scheduling is simply allocating or reserving resources to users in a communication system to maximize throughput and system efficiency. Scheduling in LTE downlink takes advantage of various factors including channel variations by allocating frequency and time resources to a user with transiently better channel conditions [5]. The quality of service requirement in a multi-user communication system varies therefore the choice of a scheduling algorithm critically impacts the system performance. These techniques are evaluated on the basis of quality of service requirement of a user, and in terms of the maximum benefit the system can derive from it using metrics of fairness, system throughput and most especially service level agreement.

\subsection{Round Robin Scheduling}

Round Robin is one of the fundamental and widely used scheduling algorithms. Its running process is very simple and easy to implement. Each active UE in a cell have equal access to resources and services at equal amount of time slots hence round robin algorithm is not a channel-dependent scheduling algorithm

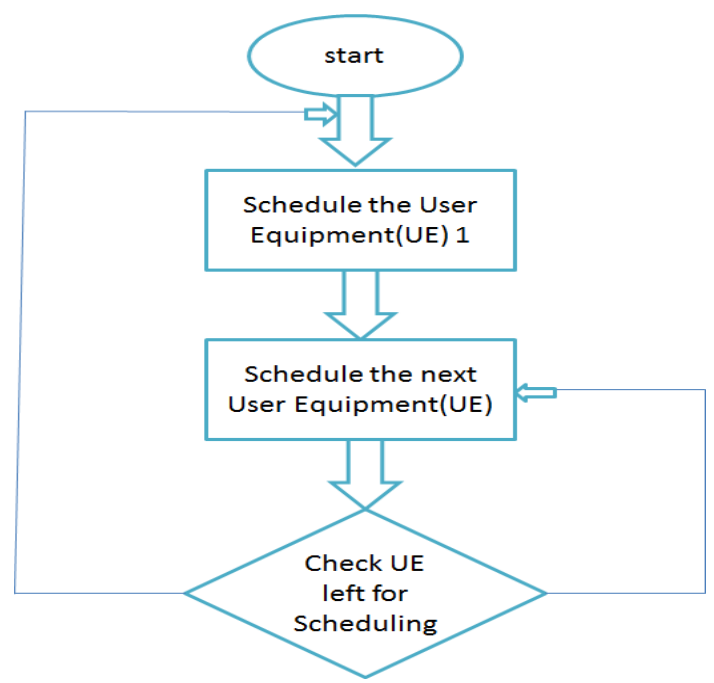

Fig-4:Round Robin Scheduling 


\section{SIMULATION RESULTS AND DISCUSSIONS}

Table-1:Simulation Parameters

\begin{tabular}{|l|l|}
\hline Parameters & Value \\
\hline $\begin{array}{l}\text { Macro cell } \\
\text { (TX power) }\end{array}$ & $20 \mathrm{dBm}$ \\
\hline $\begin{array}{l}\text { Pico cell } \\
\text { ( TX power) }\end{array}$ & $6 \mathrm{dBm}$ \\
\hline Scheduler & Round Robin \\
\hline Carrier frequency & $2 \mathrm{GHz}$ \\
\hline Bandwidth & $10 \mathrm{MHz}$ \\
\hline Bias values & 0,15 \\
\hline
\end{tabular}

Pico cell Range is increased by the bias value, which forces mobiles to perform a handover into the Pico cell, even if the received signal strength of the Pico cell is comparatively low, but this results in the interference in Range extended region.

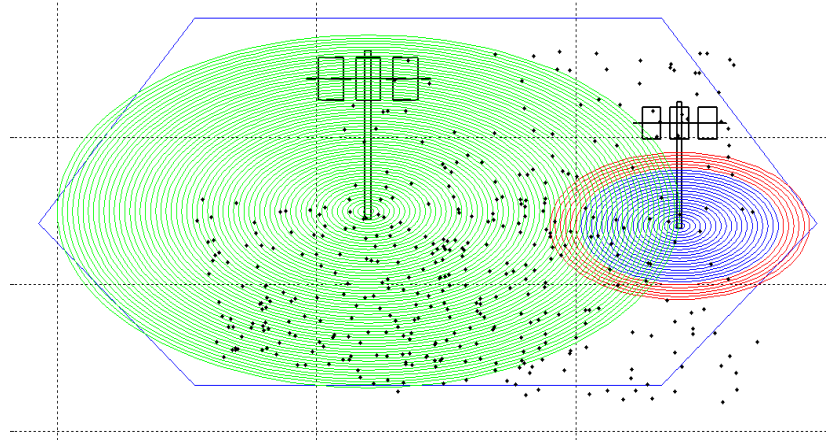

Fig-5:Scenario indicating the interference for UE in RE region

Figure 1.5 indicates the region of the two cells with range extended region of picocell being interfered by macrocell. This offloading is done various bias value, the consideration here is for $0 \mathrm{db}$ and $15 \mathrm{db}$. The performance under various load condition has been analyzed in the following section.

\subsection{Performance Under Different Load Condition}

The Figure 1.6 gives Resource usage ratio for the Users in the cell with the bias value for macrocell $(0 \mathrm{db})$, picocell(0db), All cells (Macro+pico)(0db). The result indicates that the Macrocell resource has been used maximum since there is no offloading for the user arriving in the cell. The Picocell resources has been used at the minimum due to the reduced transmitting power.

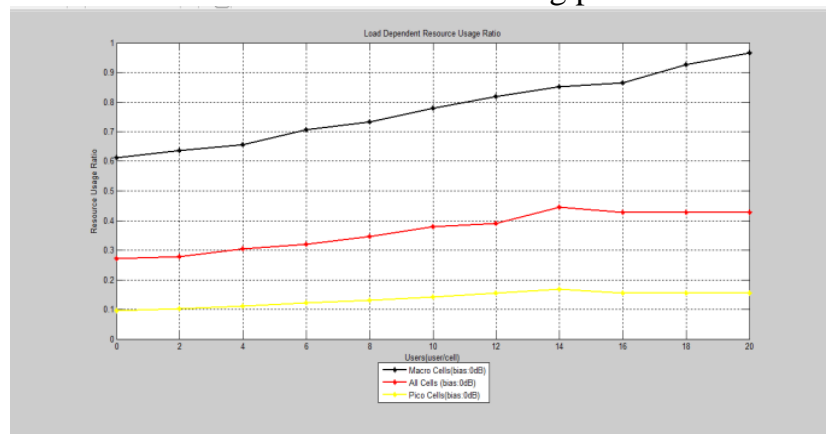

Fig-6:Resource Usage Ratio (OdB)

The Figure 1.7 gives Resource usage ratio for the Users in the cell with the bias value for macrocell $(15 \mathrm{db})$, picocell(15db), All cells (Macro+pico)(15db). The result indicates that the Picocell Resource has been used maximum since there is offloading for the user arriving in the cell. The Macrocell resources usage is reduced due to the offloading of users to picocell.

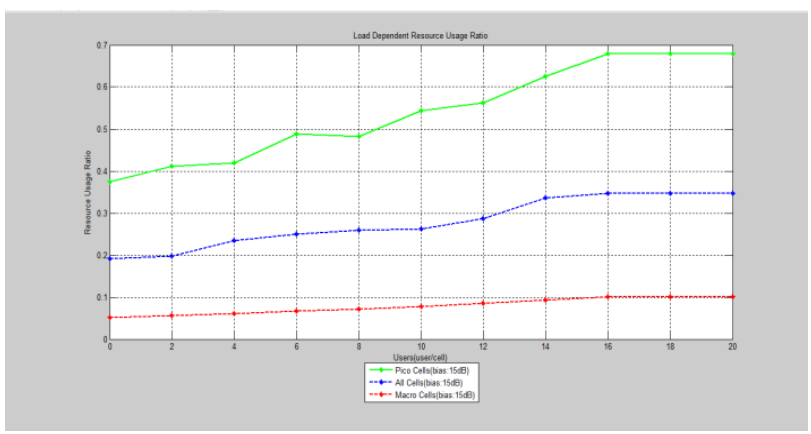

Fig-7:Resource Usage Ratio (bias-15dB)

\subsection{Throughput of Mobiles In Dependency of}

\section{Traffic Load}

Figure 1.8 gives the Mean mobile throughput for the user arrival in the cell. The Mean user throughput for the Bias value of $0 \mathrm{db}$ and $15 \mathrm{db}$ without eICIC is reduced with the user arrival rate but the round robin scheduling for eICIC throughput shows the fairness in throughput is improved with the user arrival.

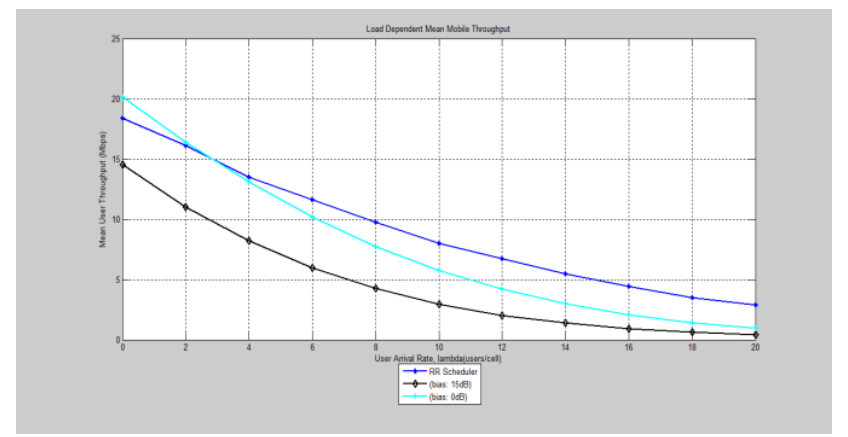

Fig-8:Mean Mobile Throughput

Figure 1.9 gives the Load dependent Cell border throughput for user arrival in the cell. The Cell border throughput for the Bias value of $0 \mathrm{db}$ and $15 \mathrm{db}$ without eICIC is reduced with the user arrival rate but the round robin scheduling for eICIC throughput shows the fairness in throughput is improved with the user arrival and the throughput for the cell border is improved using round robin. 


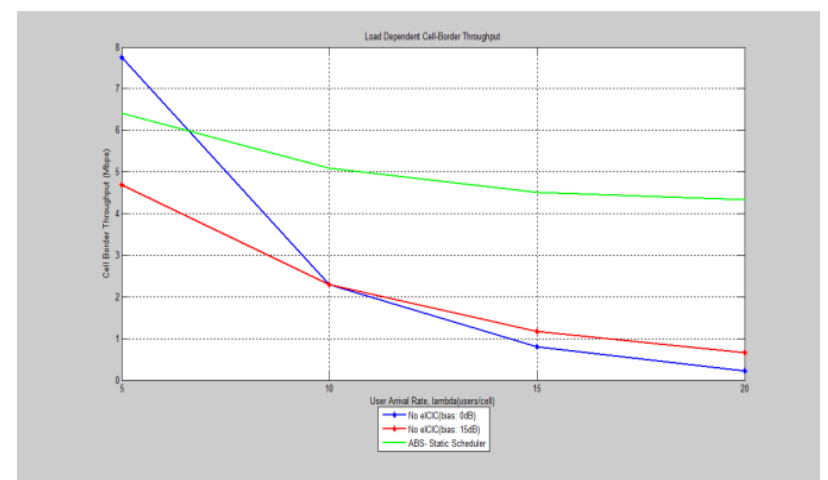

Fig-9:Cell-Border Throughput

\section{CONCLUSIONS}

The performance analysis shows that the eICIC performance for various load condition has been used to reduce the interference between the pico and macro cell. The pico layer range extension offset should be properly chosen according to the scenario, and especially be adapted to the UE distribution (i.e. load balancing).

When the range expansion scheme is applied to encourage more users to select pico cells as their serving cells by adding a positive bias, the almost blank subframe is inevitable to protect offloaded pico cell users from macro cell interference.

In this paper, Round robin scheduling has been performed in enhanced inter cell interference coordination. In future various scheduling strategies can be applied to eICIC and the performance can be improved by providing fairness and load balancing in muti cell environment.

\section{REFERENCES}

[1]Andreas Weber, Oliver Stanze. ,"Scheduling Strategies For Hetnets Using eICIC", International workshop on small cell wireless networks, 2012.

[2]Mohammed Al-Rawi. "A Dynamic Approach for Cell Range Expansion in Interference Coordinated LTEAdvanced Heterogeneous Networks", IEEE ICCS Proceedings of the 2012 .

[3]Kenta Okino, Taku Nakayama, Chiharu Yamazaki, Hirotaka Sato, and Yoshimasa Kusano. "Pico Cell Range Expansion with Interference Mitigation toward LTEAdvanced Heterogeneous Networks", IEEE International conference, Communications Workshop(ICC), 2011.

[4]]Jinyoung Oh and Youngnam Han, "Cell Selection for Range Expansion with Almost Blank Subframe in Heterogeneous Networks" IEEE 23rd International Symposium on Personal, Indoor and Mobile Radio Communications - (PIMRC), 2012.

[5]Cornel Balint, Georgeta Budura, Eugen Marza, "Scheduling Techniques Evaluation in LTE systems with Mixed Data Traffic", 2010.

[6]Yuanye Wang and Klaus I.Pedersen, "Performance Analysis of Enhanced Inter-cell Interference Coordination in LTE-Advanced Heterogeneous Networks", 2012.
[7]Andrias Racz, Norbert Reider and Gabor Fodor, "On the impact of Inter-cell Interference in LTE", IEEE "GLOBECOM" 2008.

[8]Mohammad T.Kawser, Hasib M.A.B.Farid, Abduhu R. Hasin, Adil M.J. Sadik and Ibrahim K.Razu, "Performance Comparison Between Round Robin and Proportional Fair Scheduling Methods for LTE", International Journal of Information and Electronics Engineering, Vol. 2 No. 5,September-2012.

[9]Supratim Deb, Pantelis Monogioudis, "Algorithms for Enhanced Inter-cell Interference Coordination (eICIC) in LTE HetNets", IEEE Transaction on Nerworking, 2013.

[10]Klaus I.Pedersen, Yuanye Wang, Stanislaw Stryzz, Frank Frederiksen, "Enhanced Inter-cell Interference coordination in Co-channel Multi-Layer LTE-Advanced Networks", IEEE Wireless Communication, June 2013.

[11]Stefan Schwarz, Christian Mehlf uhrer and Markus Rupp ,"Throughput Maximizing Multiuser Scheduling with Adjustable Fairness",

[12]Amitabha Ghosh, Nitin Mangalvedhe, Rapeepat Ratasuk, Bishwarup Mondal, Mark Cudak, Eugene Visotsky, and Timothy A. Thomas, "Heterogeneous Cellular Networks: from theory to practice", IEEE communications magazine, June 2012.

[13]Haidar Safa and Kamal Tohme, "LTE Uplink Scheduling Algorithms: Performance and Challenges", International Conference on Telecommunications, 2012.

[14]4G++: Advanced Performance Boosting Techniques in $4^{\text {th }}$ Generation Wireless Systems, National Telecommunication Regulatory Authority Funded Project Deliverable D4.1 